Z Rheumatol 2019· 78:499

https://doi.org/10.1007/s00393-019-0682-z

(c) Springer Medizin Verlag GmbH, ein Teil von Springer Nature 2019

Der DGRh(Deutsche Gesellschaft für Rheumatologie)-Kongress 2019 muss es sich zur Aufgabe machen, alle Rheumatologen fit für 2020 und das kommende Jahrzehnt zu machen. Die Themenschwerpunkte des Dresdner Kongresses spannen mit Systemerkrankungen und Osteoarthrose ganz bewusst einen weiten Bogen, unter dem die gesamte Rheumatologie Platz findet. Als internistischen Rheumatologen liegen uns die Systemerkrankungen mit ihren differenzialdiagnostischen Herausforderungen, breit gestreuten Organmanifestationen und der Notwendigkeit des gezielten, zeitgerechten Einsatzes von immunmodulierenden Medikamenten noch mehr am Herzen - und mit dem interdisziplinären Ansatz, auf den wir in Dresden stolz sind.

》) Systemerkrankungen sind für internistische Rheumatologen wichtige, relativ häufige Krankheitsbilder

Daher war es naheliegend, die Gelegenheit des DGRh-Kongresses zu nützen, um exemplarisch einige der Systemerkrankungen in ihrer aktuellen Entwicklung abzubilden. Ich freue mich sehr, dass ich für jedes der 4 Themen sofort eine Zusage bekommen habe - und bin allen Autorinnen und Autoren dafür dankbar. $\mathrm{Zu}$ sammen geben die 4 Beiträge einen eindrucksvollen Überblick über den internistisch-rheumatologischen Fortschritt. Unter dem Motto „Fit für 2020“ fassen sie alles Neue zusammen, das die letzten Jahre gebracht haben, und geben einen ganz prägnanten Überblick.

\title{
M. Aringer
}

Bereich Rheumatologie, Medizinische Klinik und Poliklinik III, Universitätsklinikum und Medizinische Fakultät Carl Gustav Carus, TU Dresden, Dresden, Deutschland

\section{Systemerkrankungen - Fit für 2020!}

Die prototypische Autoimmunsystemerkrankung ist noch immer der systemische Lupus erythematodes (SLE). Matthias Schneider hat mit Johanna Mucke und Rebecca Fischer-Betz aus seinem Düsseldorfer Team das Management dieser Erkrankung auf den Punkt gebracht. Noch konziser ist Fortschritt bei SLE nicht zu haben. Auch das primäre Sjögren-Syndrom als häufigste Kollagenose und immunologischer Cousin des SLE brauchte ein Update in diesem Heft, das klarerweise von Torsten Witte aus Hannover kommen musste. Auch Sjögren kurz und trotzdem umfassend geht unter diesen Bedingungen.

Neben den Kollagenosen durften auch die Vaskulitiden nicht zu kurz kommen. Die ANCA(antinukleärer zytoplasmatischer Antikörper)-assoziierten Vaskulitiden und die neuen Erkenntnisse der letzten Jahre auf diesem Gebiet fasst für uns einer der letzten DGRh-Kongresspräsidenten, Bernd Helmich aus Kirchheim unter Teck, zusammen.

Und schließlich gehört zweifellos die rheumatoide Arthritis (RA) auf die Liste der Systemerkrankungen. Auch wenn unsere Therapie die offensichtlichen Systemfolgen heute in den meisten Fällen verhindert, ist und bleibt die RA eine Systemerkrankung mit erheblichen Auswirkungen auf innere Organe. Das Thema RA wird in dieser Ausgabe im CME-Weiterbildungsbeitrag ,Therapiealgorithmus der rheumatoiden Arthritis" von Christoph Fiehn und Klaus Krüger beleuchtet. Rheumatoide Arthritis, Kollagenosen und Vaskulitiden geben gemeinsam auch einen Überblick über den Fortschritt in unseren internistisch-rheumatologischen Kerngebieten - und vermitteln so hoffentlich auch Studierenden die
Herausforderung und Faszination unseres Faches.

Neben Begeisterung und harter Arbeit machen auch Kollegialität und Freundschaften die Rheumatologie aus. Das wird einem rasch wieder bewusst, wenn man mit ein paar netten Telefonaten alle Zusagen für solch ein Heft hat. Ich habe es, seit ich als Österreicher in Sachsen und in der DGRh bin, eigentlich immer erlebt. Und ich bin sicher, dass wir dieses Zusammengehören auch auf dem Dresdner DGRh-Kongress feiern werden - mit den Kinderrheumatologen und Orthopäden und mit der Rheumaliga, die als Selbsthilfeorganisation für die Menschen eintritt, für die auch wir alle arbeiten.

Viel Spaß mit dieser Ausgabe, Willkommen in Dresden und herzliche Grüße

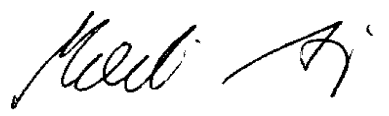

\section{Aringer}

\section{Korrespondenzadresse}

Prof. Dr. M. Aringer

Bereich Rheumatologie, Medizinische Klinik und Poliklinik III, Universitätsklinikum und Medizinische Fakultät Carl Gustav Carus, TU Dresden

Fetscherstr. 74, 01307 Dresden, Deutschland Martin.aringer@uniklinikum-dresden.de

Interessenkonflikt. M. Aringer gibt an, dass kein Interessenkonflikt besteht. 